INTERVENTIONAL CARDIOLOGY AND SURGERY

\title{
Coronary surgery with non-cardioplegic methods in patients with advanced left ventricular dysfunction: immediate and long term results
}

\author{
P E Antunes, J M Ferrão de Oliveira, M J Antunes
}

Heart 2003;89:427-43

See end of article for authors' affiliations

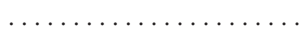

Correspondence to: Professor Manuel J Antunes, Cirurgia Cardiotorácica, Hospitais da Universidade, 3049 Coimbra Codex, Portugal; antunes.cct.huc@ mail.telepac.pt

Accepted 20 November 2002

\begin{abstract}
Objective: To evaluate perioperative results and long term survival in patients with severe left ventricular (LV) dysfunction undergoing coronary artery bypass grafting (CABG) using non-cardioplegic methods.

Methods: From April 1990 through December 1999, 4100 consecutive patients underwent isolated CABG using hypothermic ventricular fibrillation. Of these, 141 (3.4\%) had severe LV dysfunction (ejection fraction < 30\%). Mean age was $58.3(9.6)$ years. 64 patients $(45.4 \%)$ were in Canadian Cardiovascular Society class III or IV and $16(11.3 \%)$ were subjected to urgent or emergent surgery. A previous myocardial infarction was recorded in 127 (90.1\%). The majority (89.4\%) had triple vessel and $26(18.4 \%)$ had left main disease. The mean number of grafts per patient was 3.1. At least one internal thoracic artery was used in all patients and 21 (14.8\%) had bilateral internal thoracic artery grafts (1.2 arterial grafts per patient).

Results: Perioperative mortality was $2.8 \%$ (4 patients) and the incidence of acute myocardial infarction $2.8 \% .50(35.5 \%)$ patients required inotropes but only $16(11.3 \%)$ required it for longer than 24 hours; 5 patients $(3.5 \%)$ needed mechanical support. The incidence of renal failure was $3.5 \%$. Mean duration of hospital stay was 9.6 (8.3) days. Follow up was $95 \%$ complete and extended for a mean of $57(30)$ months. Late mortality was $11.5 \%$. Actuarial survival rates at 1, 3, and 5 years were $96 \%$, $91 \%$, and $86 \%$, respectively.

Conclusions: Non-cardioplegic techniques are safe and effective in preserving the myocardium during $C A B G$ in patients with coronary artery disease and poor LV function, with low operative mortality and morbidity, and encouraging medium to long term survival rates.
\end{abstract} cardioplegic methods between April 1990 and December 1999 were retrieved for analysis. Data for 141 (3.4\%) patients (group 1) with severe impairment of LV function (ejection fraction $(\mathrm{EF})<30 \%)$, determined semiquantitatively by

contrast ventriculography, were analysed. Echocardiography and nuclear ventriculography were carried out in a minority of patients and, when these additional data were available, the highest EF value was used for subsequent analysis. All patients had angina or evidence of ischaemia.

\section{Patients}

Table 1 details the perioperative clinical and angiographic data. The mean age of the 133 male $(94.3 \%)$ and eight female patients was $58.3(9.6)$ years. Forty $(28.6 \%)$ patients had diabetes and $72(51.1 \%)$ had dyslipidaemia. Sixty four $(45.4 \%)$ were in Canadian Cardiovascular Society class III or IV. Twelve $(8.5 \%)$ patients had been on intravenous antianginal medication in the last two days before the operation (unstable angina). A previous myocardial infarction was recorded in 127 patients $(90.1 \%), 11(7.8 \%)$ of which had occurred in the 30 days preceding coronary surgery. The majority $(88.7 \%)$ of the patients were operated on electively.

One hundred and twenty six patients $(89.4 \%)$ had triple and $14(9.9 \%)$ had double vessel coronary disease. Twenty six (18.4\%) had left main disease. There was only one case of repeat $\mathrm{CABG}$ among these patients. By definition, the EF was $<30 \%$ in all cases (range $12-29 \%$ ).

\section{Surgical technique and operative data}

Cardiopulmonary bypass was instituted using a bubble or membrane oxygenator and non-pulsatile flow. Patients were

Abbreviations: $C A B G$, coronary artery bypass grafting; $E F$, ejection fraction; ITA, internal thoracic artery; LV, left ventricular 
Table 1 Preoperative clinical data in 141 patients with left ventricular dysfunction

\begin{tabular}{ll}
\hline & Number \\
\hline Mean age (years) & $58.3(9.6)$ \\
Sex (male) & $133(94.3 \%)$ \\
Coronary risk factors & \\
Diabetes mellitus & $40(28.6 \%)$ \\
Smoking & $92(65.2 \%)$ \\
Hypertension & $70(49.6 \%)$ \\
Dyslipidaemia & $72(52.2 \%)$ \\
Family history of CAD & $33(23.6 \%)$ \\
History of MI & \\
Old & $116(82.3 \%)$ \\
Recent (<30 days) & $11(7.8 \%)$ \\
Peripheral vascular disease & $22(15.6 \%)$ \\
Cerebrovascular disease & $5(3.5 \%)$ \\
Angina (CCS class) & $14(9.9 \%)$ \\
I & $63(44.7 \%)$ \\
II & $46(32.6 \%)$ \\
III & $18(12.8 \%)$ \\
IV & $12(8.5 \%)$ \\
Unstable angina & $64(45.4 \%)$ \\
Cardiomegaly & $10(7.1 \%)$ \\
Anaemia & $1(0.7 \%)$ \\
Extent of disease & $14(9.9 \%)$ \\
One vessel & $126(89.4 \%)$ \\
Two vessels & $26(18.4 \%)$ \\
Three vessels & $107(75.8 \%)$ \\
Left main disease & $1(0.7 \%)$ \\
LVEDP $>20$ mm Hg & \\
Prior IABP support & \\
\hline
\end{tabular}

CAD, coronary artery disease; CCS, Canadian Cardiovascular Society; IABP, intra-aortic balloon pump; LVEDP, left ventricular end diastolic pressure; MI, myocardial infarction.

cooled to an oesophageal temperature of $30-32^{\circ} \mathrm{C}$. An LV vent was always placed through the right superior pulmonary vein and left atrium. No topical cooling was used.

The method predominantly used during the construction of the distal anastomoses was ventricular fibrillation, without clamping of the aorta. This method has been described in detail in a previous report, and we refer here to only some aspects. $^{8}$

Generally, the anastomoses to the right coronary artery or its branches on the inferior surface of the heart were constructed first, followed by the anastomoses to the branches of the circumflex system. Bypasses to the anterior descending artery and its diagonal branches were performed last. Very early in the series, the proximal anastomoses of each vein graft was usually constructed immediately after the distal one, but now all distal anastomoses are constructed first, followed by all proximal anastomoses in a single partial clamping period. In the case of ongoing ischaemia, the coronary artery branch that was likely to be involved was grafted first followed immediately by the proximal anastomosis. Control of residual and collateral blood flow was achieved by a variety of methods, mainly with the use of the coronary occluders and a gentle jet of oxygen.

Internal thoracic artery (ITA) grafts were used in all patients. In $21(14.8 \%)$ patients both left and right ITAs were used, for a mean of $1.2(0.4)$ arterial anastomoses per patient. Additionally, a mean of $1.9(0.8)$ venous anastomoses were constructed per patient. Hence, an average of 3.1 (0.7) coronary artery branches were bypassed per patient. Endarterectomies were performed in 26 cases ( $18.4 \%), 19$ of the right coronary and seven of the left system. Perfusion time was 69.4 (20.2) minutes.

\section{Statistical methods}

Data were collected prospectively on standard forms and entered into a computerised database. All data were retrospectively analysed with the assistance of Systat statistical

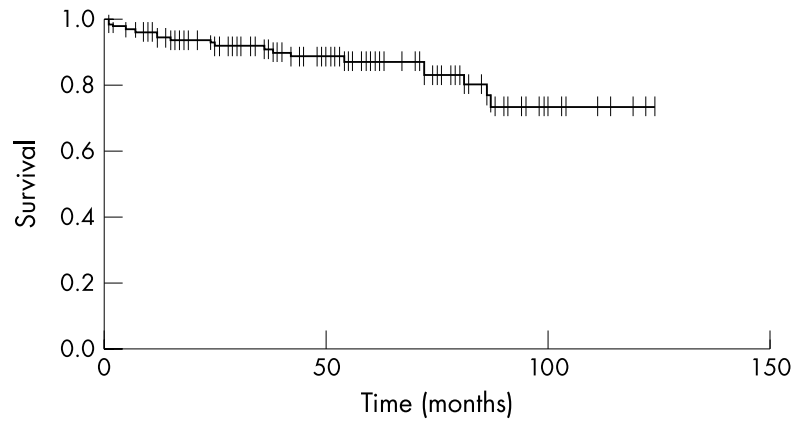

Figure 1 Kaplan-Meier survival curve of the 141 patients in the study.

software (Systat Software Inc). All data are expressed as mean (SD) or as proportions. For univariate analysis of categorical data, the $\chi^{2}$ test $(2 \times 2$ contingency tables $)$ or Fisher's exact test were used. Univariate analysis of continuous variables was carried out using Student's $t$ test. Long term actuarial survival rates were calculated using the Kaplan-Meier method.

\section{RESULTS}

There were four $(2.8 \%)$ perioperative deaths (defined as deaths in the hospital or within 30 days if the patient was discharged sooner). Three patients died of cardiac failure and one of respiratory failure. Fifty patients $(35.5 \%)$ required inotropic support (defined as the use of any inotropic agent after the patient left the operating room) but only 16 (11.3\%) for periods longer than 24 hours. Mechanical support (intra-aortic balloon or LV assist device) was used in five patients (3.5\%). Five patients $(3.5 \%)$ developed acute renal failure (creatinine $>220 \mu \mathrm{mol} / \mathrm{l})$ but none required dialysis. Four patients $(2.8 \%)$ had ECG criteria of perioperative myocardial infarction. Supraventricular arrhythmias necessitating medical or electrical treatment occurred in 24 patients ( $17.0 \%)$. Three patients $(2.1 \%)$ had an episode of ventricular fibrillation, successfully converted, and another three had transient complete atrioventricular block. Three patients $(2.1 \%)$ required re-exploration because of haemorrhage and seven (4.9\%) were reoperated for sternal dehiscence, including three with the diagnosis of acute mediastinitis (presence of pus or bacterial growth in the mediastinal tissues sampled during surgical re-exploration). Five patients (3.5\%) had a cerebrovascular accident, all with at least partial recovery. The mean length of hospital stay was 9.6 (8.3) days.

Follow up was conducted by mail or telephone and was 95\% complete. The mean follow up time of the 130 survivors (seven patients were lost) was 57 (30) months (range 9-124 months). Fifteen patients (11.5\%) had died. The actuarial survival at one, three, and five years was $96 \%, 91 \%$, and $86 \%$, respectively (fig $\mathrm{l}$ ).

To place the immediate results into perspective, results for group 1 were compared with the clinical, angiographic, and operative data, as well as of the mortality and in-hospital events, of 3557 patients with normal LV function or only mild dysfunction $(\mathrm{EF} \geqslant 40 \%$ ) who had undergone isolated CABG during the same time period (group 2; tables 2 and 3).

Compared with group 2 patients, those in group 1 were younger and had higher incidences of peripheral vascular disease, anaemia, cardiomegaly, and prior myocardial infarction. There were no significant differences in the angina symptoms or in the preoperative use of intra-aortic balloon pump.

On the other hand, patients with severe LV dysfunction had more extensive coronary artery disease (mean number of vessels involved was $2.9 \vee 2.7$ in group $2 ; \mathrm{p}<0.001$ ). This difference results essentially from a larger number of patients with single vessel disease in group 2. Hence, as would be expected, 
Table 2 Comparison of clinical and operative data between groups 1 and 2

\begin{tabular}{llll}
\hline Variable & Group 1 & Group 2 & p Value \\
\hline Number of patients & 141 & 3557 & \\
Mean age (years) & $58.3(9.6)$ & $60.1(9.2)$ & 0.032 \\
Sex (female) (\%) & 5.7 & 11.7 & 0.027 \\
Coronary risk factors (\%) & 28.6 & 20.3 & 0.017 \\
$\quad$ Diabetes mellitus & 65.2 & 53.9 & 0.008 \\
$\quad$ Smoking & 49.6 & 56.9 & $\mathrm{NS}$ \\
$\quad$ Hypertension & 52.2 & 55.5 & $\mathrm{NS}$ \\
$\quad$ Dyslipidaemia & 23.6 & 22.2 & $\mathrm{NS}$ \\
$\quad$ Family history of CAD & & & \\
History of MI (\%) & 82.3 & 45.1 & $<0.001$ \\
Old & 7.8 & 4.9 & $\mathrm{NS}$ \\
Recent (<30 days) & 15.6 & 11.1 & 0.023 \\
Peripheral vascular disease (\%) & 3.5 & 4.8 & $\mathrm{NS}$ \\
Cerebrovascular disease (\%) & 46.1 & 42.0 & $\mathrm{NS}$ \\
Angina (CCS class III/IV) & 45.4 & 7.9 & $<0.001$ \\
Cardiomegaly (\%) & 7.1 & 4.2 & 0.042 \\
Anaemia (\%) & $2.9(0.3)$ & $2.7(0.6)$ & $<0.001$ \\
Number of diseased vessels (mean) & 18.4 & 15.8 & $\mathrm{NS}$ \\
Left main disease (\%) & 89.3 & 92.1 & $\mathrm{NS}$ \\
Surgical priority (elective) (\%) & $3.1(0.7)$ & $2.8(0.8)$ & $<0.001$ \\
Number of grafts/patient (mean) & $1.2(0.5)$ & $1.3(0.5)$ & $\mathrm{NS}$ \\
Number of arterial grafts/patient (mean) & $1.9(0.8)$ & $1.5(0.8)$ & $<0.001$ \\
Number of venous grafts/patient (mean) & 100.0 & 99.2 & $\mathrm{NS}$ \\
Arterial conduits (\%) & 14.8 & 24.1 & $<0.001$ \\
$\quad$ IITA & 18.4 & 9.2 & $<0.001$ \\
RITA & $69.4(20.2)$ & $63.2(26.0)$ & $<0.001$ \\
Endarterectomy (\%) & & \\
Perfusion time (min) (mean) & & & \\
\hline LITA, left internal mammary artery; RITA, right internal mammary artery. & & \\
\hline
\end{tabular}

\begin{tabular}{|lccc|}
\hline Table 3 & Comparison of results (\%) between groups 1 and 2 & \\
\hline Variable & Group 1 & Group 2 & p Value \\
\hline Mortality & 2.8 & 0.9 & 0.025 \\
Morbidity & 35.5 & 5.5 & $<0.001$ \\
Inotropic support & 3.5 & 0.6 & $<0.001$ \\
Mechanical support & 2.8 & 4.1 & $\mathrm{NS}$ \\
Perioperative Ml & 3.5 & 2.8 & $\mathrm{NS}$ \\
Acute renal failure & 2.1 & 2.5 & $\mathrm{NS}$ \\
Reoperation for bleeding & 4.9 & 2.1 & 0.022 \\
Reoperation for sternal complications & 3.5 & 2.1 & $\mathrm{NS}$ \\
Stroke & 19.1 & 23.3 & $\mathrm{NS}$ \\
Arrhythmias & $9.6(8.3)$ & $7.9(5.6)$ & 0.002 \\
Hospital stay (mean time in days) & \multicolumn{3}{l}{} \\
\hline
\end{tabular}

there was a significant difference in the number of coronary artery grafts constructed per patient $(3.2 \vee 2.8$, respectively; $\mathrm{p}<0.0001)$. The mean number of arterial grafts per patient was similar in the two groups.

Mortality was higher in group $1(2.8 \%)$ than in group 2 $(0.9 \% ; p=0.025)$. The use of inotropes or mechanical support and the number of sternal complications were also higher in group 1. There were no significant differences in the incidences of myocardial infarction, stroke, acute renal failure, arrhythmias, and reoperation for bleeding. Finally, patients in group 1 had a longer hospital stay ( 1.7 days longer; $p=0.002$ ) but there was no difference if only survivors were counted.

\section{DISCUSSION}

Patients with coronary disease and advanced LV dysfunction have a poor prognosis when treated only medically. They have a limited survival and usually die of cardiac causes. On the other hand, several studies have shown that they respond better to coronary surgery. ${ }^{12410}$ Although transplantation is an effective treatment option, it is limited by availability of donor organs and by the implications of immunosuppression. Therefore, revascularisation should always be considered as an option in these patients. It has been shown that CABG increases survival, improves functional status, provides better control of angina symptoms, and reduces the prevalence of sudden cardiac death caused by arrhythmias. ${ }^{1347911}$

\section{Case selection and myocardial protection}

One important question to be addressed is which patients with severe coronary artery disease and low EF would derive most benefit. It has been suggested that symptoms of angina (versus symptoms of heart failure) are associated with a more favourable outcome. ${ }^{12}$ Some groups require evidence of reversible ischaemia or viability of hibernating myocardium by radioisotopic scanning, positron emission tomography, or dobutamine echocardiography to select patients for this approach. ${ }^{13}{ }^{14}$ We have not excluded patients because of lack of significant angina, nor have we routinely used viability studies to select candidates for grafting. Patients were considered to be unsuitable for grafting only if they had angiographic evidence of diffuse distal disease, particularly of the anterior descending artery, or in the presence of comorbidities that significantly increase operative risk.

We concur with others in that, in patients with little functional reserve, favourable results can only be achieved 
with optimal myocardial protection. An unending series of reports dealing with cardioplegia in any of its numerous variations have shown this unequivocally. However, this evidence does not mean that non-cardioplegic methods result in less protection. In fact, several randomised clinical studies have shown that intermittent aortic cross clamping provides protection that is equivalent to, if not better than, blood cardioplegia and preserves LV diastolic function better. ${ }^{15-18}$ We and others have previously shown the safety and efficacy of these methods, even in higher risk patients, possibly because during the operation the myocardium is continuously perfused with the patient's unaltered blood. ${ }^{8}{ }^{19-21}$

The use of off-pump coronary bypass in this subgroup of patients has recently been studied with respect to early outcomes. Two recent retrospective studies have reported similar results when comparing off-pump coronary bypass with cardioplegic techniques and concluded that this method is safe and efficacious. ${ }^{22}{ }^{23}$ The question may also be raised about non-cardioplegic methods. Nonetheless, anatomical factors, which were frequently found in our patients, such as cardiomegaly and, more important, heavily calcified vessels requiring endarterectomy, may preclude off-pump coronary bypass in many of these patients. We believe that longer follow up is required before a conclusion can be reached.

\section{Perioperative results and survival}

Recent reports indicate that the perioperative mortality after CABG in patients with advanced LV dysfunction varies between $2.5-8 \% .^{5-8}$ In the present series, the perioperative mortality was $2.8 \%$. As others have experienced, we have had a higher requirement for inotropic support than in our patients with an EF $>40 \%$. However, in contrast with other studies, in our study very few patients $(3.5 \%)$ required mechanical support in the early postoperative period, and the incidence of myocardial infarction was $2.8 \%{ }^{59}{ }^{12}$ Considering the characteristics of the group, these results are quite acceptable and compare favourably with those of series where cardioplegic methods were used. The relatively low incidence of other causes of morbidity and the short hospital stay also constitute good clinical benchmarks for a relatively smooth perioperative period.

However, the incidence of sternal complications was somewhat higher $(4.9 \%)$ than those reported by others, which may be related to the fact that all of our patients received ITA grafts, including $14.8 \%$ in whom both ITAs were used. ${ }^{6}$ The use of ITA grafts in this cohort of patients is an important issue because the reports are conflicting. Concerns about the short term limitations of ITA grafts may have made surgeons more likely to use venous conduits in these high risk patients. Eventual problems of the ITA include lower initial flow than with vein grafts, the potential for spasm, the time required for harvesting in unstable patients, and, eventually, the inability to deliver the cardioplegic solution down a pedicled arterial graft. Additionally, the reduced late survival usually associated with LV dysfunction, advanced age, and significant comorbidities in these patients may offset the survival advantage generally associated with ITA use.

Despite these concerns, we follow a more liberal approach. We used left ITA grafts in all patients of the study group and, additionally, right ITA grafts in $21(14.8 \%)$ patients. Some similar studies reported left ITA use in $76-86 \%$ of patients with LV dysfunction, with excellent initial results. ${ }^{71}$ Anderson and colleagues ${ }^{24}$ reported the use of left ITA in $70 \%$ of patients with ventricular dysfunction presenting with chronic congestive heart failure and concluded that its use enhanced late survival. By contrast, others have used ITA in as few as $41 \%$ of patients, and some question the survival benefit of the use of the left ITA conduit in these patients. ${ }^{25}{ }^{26}$ In any case, early outcomes have not been adversely affected by the use of arterial grafts in the setting of ventricular dysfunction.
In a previous publication, we had already shown this favourable immediate outcome. Apart from increasing the number of patients studied, we have now been able to complete the medium term follow up (mean 57 (30) months). ${ }^{8}$ Our five year survival of $86 \%$ compares favourably with those reported by others after CABG in patients with severe LV dysfunction of between $60-80 \% .^{79^{11}}{ }^{27-29}$ However, it is important to note that some of these studies included only patients with an EF of $\leqslant 20 \%$. Another possible reason for this difference may be related to our population being somewhat younger and with fewer female patients, which are two important clinical factors positively correlated with early and late death. Compared with the dismal outcome generally observed with medical treatment alone, these survival rates have to be considered excellent.

The present study has some limitations. These include the definition of severe LV dysfunction as EF of $<30 \%$ determined semiquantitatively by contrast ventriculography, which is not very precise. In fact, assessing ventricular function on contrast ventriculography is not entirely satisfactory. In some cases this study was done after the coronary arteriogram, which could have overestimated the incidence of LV dysfunction. However, in many cases where there was doubt, other methods of indirect measurement of the EF helped to clarify the situation. Additionally, because the long term survival results in the group with $\mathrm{EF} \geqslant 40 \%$ are not available yet, it is not possible to put into perspective these results by comparison with those here reported for patients with ventricular dysfunction. Finally, although some of the significant differences found between groups 1 and 2 may have little clinical relevance, it is quite evident that the higher incidence of diabetes and the more extensive vessel disease, requiring a higher number of grafts, may have had an impact in the early and long term results.

In conclusion, the results reported herein confirm that non-cardioplegic techniques are safe and effective in preserving the myocardium during $\mathrm{CABG}$, including in patients with poor LV function, in whom low operative mortality and morbidity rates were achieved. This study also confirmed an encouraging medium to long term survival in this high risk population.

\section{Authors' affiliations}

P E Antunes, J M Ferrão de Oliveira, M J Antunes, Cardiothoracic Surgery, University Hospital, Coimbra, Portugal

\section{REFERENCES}

1 Alderman EL, Fisher LD, Litwan P, et al. Results of coronary artery surgery in patients with poor left ventricular function (CASS). Circulation 1983:68:785-95

2 Zubiate P, Kay JH, Mendez AM. Myocardial revascularization for the patient with drastic impairment of function of the left ventricle. J Thorac Cardiovasc Surg 1977;73:84-6.

3 Scott SM, Deupree RH, Sharma GVRK, et al. VA study of unstable angina. 10-year results show duration of surgical advantage for patients with impaired ejection fraction. Circulation 1994;90(suppl 2):120-3.

4 Pigott JD, Kouchoucos NT, Oberman A, et al. Late results of surgical and medical therapy for patients with coronary artery disease and depressed left ventricular function. J Am Coll Cardiol 1985;5:1036-45.

5 Trachiotis GD, Weintraub WS, Johnston T, et al. Coronary artery bypass grafting in patients with advanced left ventricular dysfunction. Ann Thorac Surg 1998;66:1632-9

6 Mickleborough LL, Carson S, Tamariz M, et al. Results of revascularization in patients with severe left ventricular dysfunction. I Thorac Cardiovasc Surg 2000;1 19:550-7.

7 Elefteriades JA, Kron IL. CABG in advanced left ventricular dysfunction. Cardiol Clin 1995; 13:35-42.

8 Antunes PE, Oliveira JF, Antunes M. Non-cardioplegic coronary surgery in patients with severe left ventricular dysfunction. Eur J Cardiothorac Surg 1999; 16:331-6.

9 Kaul TK, Agnihotri AK, Fields BL, et al. Coronary artery bypass grafting in patients with an ejection fraction of twenty percent or less. J Thorac Cardiovasc Surg 1996;66:1632-9.

10 Luciani GB, Faggian G, Razzaolini R, et al. Severe ischemic left ventricular failure: coronary operation or heart transplantation? Ann Thorac Surg 1993;55:719-23. 
11 Mickleborough LL, Maruyama H, Takagi $Y$, et al. Results of revascularization in patients with severe left ventricular dysfunction. Circulation 1995;92(suppl 2):73-9.

12 Lansman SL, Cohen M, Galla JD, et al. Coronary bypass with ejection fraction of 0.20 or less using centigrad cardioplegia: long term follow-up. Ann Thorac Surg 1993;56:480-6.

13 DiCarli MF, Maddahi J, Rokhsar S, et al. Long-term survival of patients with coronary artery disease and left ventricular dysfunction: implications for the role of myocardial viability assessment in management decisions. J Thorac Cardiovasc Surg 1999:1 16:997-1004.

14 Afridi I, Grayburn PA, Panza JA, et al. Myocardial viability during dobutamine echocardiography predicts survival in patients with coronary artery disease and severe left ventricular systolic dysfunction. J Am Coll Cardiol 1998;32:921-6.

15 Anderson JR, Hossein-Nia M, Kallis $\mathrm{P}$, et al. Comparison of two strategies for myocardial management during coronary artery operations. Ann Thorac Surg 1994;58:768-73.

16 Gerola LR, Oliveira SA, Moreira LP, et al. Blood cardioplegia with warm reperfusion versus intermittent aortic cross clamping in myocardial revascularization. J Thorac Cardiovasc Surg 1993;106:491-6.

17 Musumeci F, Feccia M, MacCarthy PA, et al. Prospective randomized trial of single clamp technique versus intermittent ischemic arrest: myocardial and neurological outcome. Eur J Cardi-thorac Surg 1998;13:702-9

18 Casthely PA, Shah C, Mekhjiam H, et al. Left ventricular diastolic function after coronary artery bypass grafting: a correlative study with three different myocardial protection techniques. J Thorac Cardiovasc Surg 1997; 114:254-60.

19 Antunes MJ, Bernardo JB, Oliveira JM, et al. Coronary artery bypass surgery with intermittent aortic cross-clamping. Eur J Cardiothorac Surg 1992:6:189-94.
20 Bonchek LI, Burlingame MW, Vazales BE, et al. Applicability of noncardioplegic coronary bypass to high-risk patients: selection of patients, technique, and clinical experience in 3000 patients. J Thorac Cardiovasc Surg 1992;103:230-7.

21 Akins CW. Hypothermic fibrillatory arrest for coronary artery bypass grafting. J Card Surg 1992;7:342-7.

22 Arom KV, Flavin TF, Emery RW, et al. Is low ejection fraction safe for of-pump coronary bypass operation? Ann Thorac Surg 2000;70:1021-5.

23 Yokoyama T, Baumgartner FJ, Gheissari A, et al. Off-pump versus on-pump coronary bypass in high-risk subgroups. Ann Thorac Surg 2000;70: 1546-50

24 Anderson WA, Ilkowski DA, Mahan VL, et al. Coronary artery bypass grafting in patients with chronic congestive heart failure: 10-years experience with 203 patients. J Card Surg 1997:12:167-75.

25 Baumgartner FJ, Omari BO, Goldberg S, et al. Coronary artery bypass grafting patients with profound ventricular dysfunction. Tex Heart Inst J 1998;25: 125-9.

26 Canver CC, Heisey DM, Nichols RD, et al. Long-term survival benefit of internal thoracic artery grafting is negligible in a patient with bad ventricle. J Cardiovasc Surg (Torino) 1998;39:57-63.

27 Battista G, Montalbano G, Casali G, et al. Predicting long term functional results after myocardial revascularization in ischemic cardiomyopathy. J Thorac Cardiovasc Surg 2000;120:478-89.

28 Lorusso R, Canna GL, Ceconi C, et al. Long-term results of coronary artery bypass grafting procedure in the presence of left ventricular dysfunction and hibernating myocardium. Eur J Cardiothorac Surg 2001:20:937-48.

29 Bouchart F, Tabley A, Litzler PY, et al. Myocardial revascularization in patients with severe ischemic left ventricular dysfunction. Long term follow-up in 141 patients. Eur J Cardiothorac Surg 2001;20:1157-62.

\section{ELECTRONIC PAGES}

\section{eHEART: www.heartinl.com}

ju

he following electronic only articles are published in conjunction with this issue of Heart.

Successful radiofrequency catheter ablation of "clockwise" and "counterclockwise" bundle branch re-entrant ventricular tachycardia in the absence of myocardial or valvar dysfunction without detecting bundle branch potentials

K Matsuoka, E Fujii, F Uchida

A case is reported of a patient with only isolated conduction abnormalities of the His-Purkinje system with no identifiable myocardial or valvar dysfunction, leading to "clockwise" and "counterclockwise" bundle branch re-entrant ventricular tachycardias (BBRVTs). The electrophysiological study showed infra-Hisian conduction system disease and two different inducible wide QRS complex tachycardias. Neither right bundle branch nor left bundle branch potentials were recorded despite extensive catheter manipulation. However, these tachycardias were diagnosed as BBRVTs by using entrainment manoeuvres and comparing the HV intervals during both sinus rhythm and the tachycardias. These tachycardias were eliminated by catheter ablation of the right bundle branch, using the morphology of the local electrograms and anatomical findings.

(Heart 2003;89:el2) www.heartjnl.com/cgi/content/full/89/ $4 / \mathrm{e} 12$

\section{Concordance for hypoplastic left heart syndrome in a monochorionic twin pregnancy}

\section{R E Andrews, A C Cook, R W M Yates}

The risk of structural heart disease is significantly higher in twin pregnancies than in singleton pregnancies, but the concordance rate has been found to be relatively low, even in monochorionic pregnancies. This is the first report of a monochorionic twin pregnancy concordant for hypoplastic left heart syndrome (HLHS), the diagnosis having been made by fetal echocardiography at 15 weeks' gestation. The findings were confirmed at necropsy at 17 weeks' gestation, following termination of pregnancy. Both twins had mitral and aortic atresia, with severely hypoplastic aortic arches. This report adds weight to there being a genetic component to the cause of HLHS in some cases and illustrates how the findings from early fetal echocardiography with postmortem follow up can help to extend the understanding of the aetiology of this condition.

(Heart 2003;89:el3) www.heartjnl.com/cgi/content/full/89/ $4 / \mathrm{e} 13$

Toad venom poisoning: resemblance to digoxin toxicity and therapeutic implications

R M Gowda, R A Cohen, I A Khan

A healthy man developed gastrointestinal symptoms after ingesting purported aphrodisiac pills. He had severe unrelenting bradycardia, hyperkalaemia, and acidosis. He rapidly developed severe life threatening cardiac arrhythmias and died after a few hours. He was found to have positive serum digoxin concentrations, although he was not taking digoxin. Toad venom poisoning is similar to digitalis toxicity and carries a high mortality. Cardiac glycoside poisoning can occur from ingestion of various plants and animal toxins, and the venom gland of cane toad (Bufo marinus) contains large quantities of cardiac glycosides. Toad venom, a constituent of an aphrodisiac, was considered responsible for the development of clinical manifestations and death in this patient. Digoxin specific Fab fragment has been reported to be beneficial in the treatment of toad venom poisoning. This report alerts physicians to the need to be aware of a new community toxic exposure, as prompt treatment with digoxin specific Fab fragment may be life saving. The treatment approach to patients with suspected toad venom poisoning is described

(Heart 2003;89:el4) www.heartjnl.com/cgi/content/full/89/ $4 / \mathrm{e} 14$ 